\title{
A generalized regularization theorem and Kawashima's relation for multiple zeta values
}

\author{
Masanobu Kaneko, Ce Xu, and Shuji Yamamoto
}

December 1, 2020

IIIII

\begin{abstract}
Kawashima's relation is conjecturally one of the largest classes of relations among multiple zeta values. Gaku Kawashima introduced and studied a certain Newton series, which we call the Kawashima function, and deduced his relation by establishing several properties of this function. We present a new approach to the Kawashima function without using Newton series. We first establish a generalization of the theory of regularizations of divergent multiple zeta values to Hurwitz type multiple zeta values, and then relate it to the Kawashima function. Via this connection, we can prove a key property of the Kawashima function to obtain Kawashima's relation.
\end{abstract}

\section{Introduction}

For integers $k_{1}, \ldots, k_{r}$ with $k_{r}>1$ and a parameter $x$ (a real number with $|x|<1$ ), let us consider the multiple zeta value of Hurwitz type

$$
\zeta^{(x)}\left(k_{1}, \ldots, k_{r}\right):=\sum_{0<m_{1}<\cdots<m_{r}} \frac{1}{\left(m_{1}+x\right)^{k_{1}} \cdots\left(m_{r}+x\right)^{k_{r}}} .
$$

In this paper, we first discuss in $\$ 2$ two regularizations, the series and the integral regularization, of $\zeta^{(x)}\left(k_{1}, \ldots, k_{r}\right)$ when $k_{r}=1$, and establish a connection between the two, generalizing the well-known regularization theorem for classical multiple zeta values (see for instance [2]).

As an application, we shall give in $\$ 3$ a different approach to Kawashima's relation of multiple zeta values. Instead of using the Newton series ([6]), we define the Kawashima function $F(\mathbf{k} ; x)$ for each index $\mathbf{k}$ by a Taylor series ([3.1) in 93$)$ and establish a formula expressing $F(\mathbf{k} ; x)$ in terms of stuffle-regularized polynomials (Theorem 3.1). This enables us to deduce easily the stuffle product property (3.2) of $F(\mathbf{k} ; x)$ and thereby obtain Kawashima's relation (Corollary 3.2).

\section{Regularizations}

In this section, we briefly review the theory of regularization of multiple zeta values, and extend the theory to Hurwitz type multiple zeta values (1.1).

\footnotetext{
AMS Subject Classification: $11 \mathrm{M} 32$

Keywords: multiple zeta value, Hurwitz multiple zeta value, regularization, Kawashima function, Kawashima's relation

This work was supported by JSPS KAKENHI JP16H06336, JP18K03221, JP18H05233.
} 
For any index set $\mathbf{k}=\left(k_{1}, \ldots, k_{r}\right) \in \mathbb{N}^{r}$, we consider two quantities

$$
\zeta_{N}^{(x)}(\mathbf{k})=\zeta_{N}^{(x)}\left(k_{1}, \ldots, k_{r}\right):=\sum_{0<m_{1}<\cdots<m_{r}<N} \frac{1}{\left(m_{1}+x\right)^{k_{1}} \cdots\left(m_{r}+x\right)^{k_{r}}}
$$

and

$$
\mathrm{Li}_{\mathbf{k}}^{(x)}(t)=\mathrm{Li}_{k_{1}, \ldots, k_{r}}^{(x)}(t):=\sum_{0<m_{1}<\cdots<m_{r}} \frac{t^{m_{r}+x}}{\left(m_{1}+x\right)^{k_{1}} \cdots\left(m_{r}+x\right)^{k_{r}}},
$$

where $N$ is a fixed positive integer and $t$ is another real parameter with $0<t<1$. If $r=0$, in which case we denote $\mathbf{k}=\varnothing$, these are understood as $\zeta_{N}^{(x)}(\varnothing)=\operatorname{Li}_{\varnothing}^{(x)}(t)=1$. These quantities are finite, but diverge if $k_{r}=1$ as $N \rightarrow \infty$ or $t \rightarrow 1$ respectively. The following proposition says that there exist polynomials describing the divergence.

Proposition 2.1. For each index set $\mathbf{k}$, there uniquely exist polynomials $Z_{*}^{(x)}(\mathbf{k} ; T)$ and $P^{(x)}(\mathbf{k} ; T)$ in $\mathbb{R}[T]$ characterized by

$$
\zeta_{N}^{(x)}(\mathbf{k})=Z_{*}^{(x)}(\mathbf{k} ; \log N-\psi(1+x))+O\left(N^{-1} \log ^{p} N\right) \quad \text { as } N \rightarrow \infty \quad(\exists p>0)
$$

and

$$
\mathrm{Li}_{\mathbf{k}}^{(x)}(t)=P^{(x)}(\mathbf{k} ;-\log (1-t))+O\left((1-t) \log ^{p}(1-t)\right) \quad \text { as } t \rightarrow 1 \quad(\exists p>0) .
$$

Here, $\psi(1+x)$ is the digamma function $\Gamma^{\prime}(1+x) / \Gamma(1+x)$.

Proof. The uniqueness is clear if exist, because a polynomial in $\log N-\psi(1+x)$ (resp. in $-\log (1-t)$ ) which converges to 0 when $N \rightarrow \infty$ (resp. $t \rightarrow 1$ ) is identically zero.

As for the existence of $Z_{*}^{(x)}(\mathbf{k} ; T)$, we may proceed algebraically as in the classical case $x=0$. First, when $\mathbf{k}=\varnothing$, we have $Z_{*}^{(x)}(\varnothing ; T)=P^{(x)}(\varnothing ; T)=1$ by convention. Noting that $\zeta_{N}^{(x)}(\mathbf{k})$ obeys the standard stuffle product rule $\zeta_{N}^{(x)}(\mathbf{k}) \zeta_{N}^{(x)}(\mathbf{l})=\zeta_{N}^{(x)}(\mathbf{k} * \mathbf{l})$ (we extend by linearity the symbol $\zeta_{N}^{(x)}$ to the formal sum of indices), we may write $\zeta_{N}^{(x)}(\mathbf{k})$ as a polynomial in $\zeta_{N}^{(x)}(1)$ with coefficients which are $\mathbb{Q}$-linear combinations of $\zeta_{N}^{(x)}(\mathbf{l})$ 's with admissible $\mathbf{l}$ (an index set is called admissible if the last entry is greater than 1). For the precise definition of the stuffle (sometimes called harmonic) product $\mathbf{k} * \mathbf{l}$, see for instance [5]. Then the polynomial $Z_{*}^{(x)}(\mathbf{k} ; T)$ is obtained by replacing $\zeta_{N}^{(x)}(1)$ by $T$ and the coefficients by the same linear combinations of $\zeta^{(x)}(\mathbf{l})$ instead of $\zeta_{N}^{(x)}(\mathbf{l})$. Here we note the asymptotic behavior of $\zeta_{N}^{(x)}(1)$,

$$
\sum_{n=1}^{N-1} \frac{1}{n+x}=\log N-\psi(1+x)+O\left(N^{-1}\right) \quad(N \rightarrow \infty),
$$

which is easily obtained from the well-known

$$
\psi(1+x)=-\gamma+\sum_{n=1}^{\infty}\left(\frac{1}{n}-\frac{1}{n+x}\right) \quad(\gamma: \text { Euler's constant })
$$

and

$$
\sum_{n=1}^{N-1} \frac{1}{n}=\log N+\gamma+O\left(N^{-1}\right), \quad \sum_{n=N}^{\infty}\left(\frac{1}{n}-\frac{1}{n+x}\right)=O\left(N^{-1}\right) \quad(N \rightarrow \infty) .
$$

Also we use the (easy) estimate

$$
\zeta_{N}^{(x)}(\mathbf{l})=\zeta^{(x)}(\mathbf{l})+O\left(N^{-1} \log ^{p} N\right) \quad \text { as } N \rightarrow \infty \quad(\exists p>0)
$$


for admissible $\mathbf{l}$.

As for the existence of $P^{(x)}(\mathbf{k} ; T)$, the algebraic method using the standard (integral) shuffle

product appears not applicable because $\mathrm{Li}_{\mathbf{k}}^{(x)}(t)$ does not satisfy the shuffle product rule when $x \neq 0$. However, we may obtain a concrete form of $P^{(x)}(\mathbf{k} ; T)$ by using the iterated integral expression of $\operatorname{Li}_{\mathbf{k}}^{(x)}(t)$ :

$$
\begin{gathered}
\operatorname{Li}_{\mathbf{k}}^{(x)}(t)=\int_{0<u_{1}<\cdots<u_{k}<t} \frac{u_{1}^{x} d u_{1}}{1-u_{1}} \underbrace{\frac{d u_{2}}{u_{2}} \cdots \frac{d u_{k_{1}}}{u_{k_{1}}}}_{k_{1}-1} \frac{d u_{k_{1}+1}}{1-u_{k_{1}+1}} \underbrace{\frac{d u_{k_{1}+2}}{u_{k_{1}+2}} \cdots \frac{d u_{k_{1}+k_{2}}}{u_{k_{1}+k_{2}}}}_{k_{2}-1} \cdots \\
\cdots \frac{d u_{k-k_{r}}}{u_{k-k_{r}}} \frac{d u_{k-k_{r}+1}}{1-u_{k-k_{r}+1}} \underbrace{\frac{d u_{k-k_{r}+2}}{u_{k-k_{r}+2}} \cdots \frac{d u_{k}}{u_{k}}}_{k_{r}-1},
\end{gathered}
$$

where $k=k_{1}+\cdots+k_{r}$. We postpone the actual computation to the proof of Proposition 2.3 , formula (2.15) (of course there is no circular argument).

Recall the $\mathbb{R}$-linear map $\rho$ from $\mathbb{R}[T]$ to itself defined via the identity

$$
\rho\left(e^{T y}\right)=A(y) e^{T y}
$$

in the formal power series algebra $\mathbb{R}[T][[y]]$ on which $\rho$ acts coefficientwise, where

$$
A(y)=e^{\gamma y} \Gamma(1+y)=\exp \left(\sum_{n=2}^{\infty} \frac{(-1)^{n}}{n} \zeta(n) y^{n}\right) \in \mathbb{R}[[y]] .
$$

This map $\rho$ plays a crucial role in the theory of regularization of multiple zeta values (see [2]). We extend the fundamental theorem (Theorem 1 in [2]) to our setting.

Theorem 2.2. For any index set $\mathbf{k}$, we have

$$
P^{(x)}(\mathbf{k} ; T)=\rho\left(Z_{*}^{(x)}(\mathbf{k} ; T-\gamma-\psi(1+x))\right) .
$$

When $x=0$, the polynomial $Z_{*}^{(x)}(\mathbf{k} ; T-\gamma-\psi(1+x))$ becomes the usual stuffle regularized polynomial $Z_{*}(\mathbf{k} ; T)$ (recall $\psi(1)=-\gamma$ ), and $P^{(0)}(\mathbf{k} ; T)$ equals the shuffle regularized polynomial $Z_{\mathrm{II}}(\mathbf{k} ; T)$. Thus the theorem is a generalization of the fundamental theorem $Z_{\text {II }}(\mathbf{k} ; T)=\rho\left(Z_{*}(\mathbf{k} ; T)\right)$ of regularization in the theory of usual multiple zeta values (see [2, 5]).

We prove Theorem 2.2 by establishing the generating function identities as follows. We treat the case $\mathbf{k}=(1, \ldots, 1)$ separately. For non-empty index sets $\mathbf{k}=\left(k_{1}, \ldots, k_{r}\right)$ and $\mathbf{l}=\left(l_{1}, \ldots, l_{s}\right)$, we write $\mathbf{k}_{+}=\left(k_{1}, \ldots, k_{r-1}, k_{r}+1\right)$ and

$$
\mathbf{k} \circledast \mathbf{l}=\left(\left(k_{1}, \ldots, k_{r-1}\right) *\left(l_{1}, \ldots, l_{s-1}\right), k_{r}+l_{s}\right) .
$$

Moreover, $\mathbf{l}^{\star}$ denotes the formal sum of $2^{s-1}$ index sets of the form $\left(l_{1} \square \cdots \square l_{s}\right)$, where ' + ' or ', ' is inserted in each $\square$. Then we have

$$
\zeta^{(x)}\left(\mathbf{k} \circledast \mathbf{l}^{\star}\right)=\sum_{0<m_{1}<\cdots<m_{r}=n_{s} \geq \cdots \geq n_{1}>0} \frac{1}{\left(m_{1}+x\right)^{k_{1}} \cdots\left(m_{r}+x\right)^{k_{r}}\left(n_{1}+x\right)^{l_{1}} \cdots\left(n_{s}+x\right)^{l_{s}}} .
$$

Proposition 2.3. 1) For $Z_{*}^{(x)}$, we have the identities

$$
\sum_{m=0}^{\infty} Z_{*}^{(x)}(\underbrace{1, \ldots, 1}_{m} ; T) y^{m}=\frac{\Gamma(1+x) e^{\psi(1+x) y}}{\Gamma(1+x+y)} \cdot e^{T y}
$$


and

$$
\sum_{m=0}^{\infty} Z_{*}^{(x)}(\mathbf{k}_{+}, \underbrace{1, \ldots, 1}_{m} ; T) y^{m}=\sum_{m=0}^{\infty}(-1)^{m} \zeta^{(x)}\left(\mathbf{k} \circledast(\underbrace{1, \ldots, 1}_{m+1})^{\star}\right) y^{m} \cdot \frac{\Gamma(1+x) e^{\psi(1+x) y}}{\Gamma(1+x+y)} \cdot e^{T y}
$$

for non-empty index set $\mathbf{k}$.

2) For $P^{(x)}$, we have

$$
\sum_{m=0}^{\infty} P^{(x)}(\underbrace{1, \ldots, 1}_{m} ; T) y^{m}=\frac{\Gamma(1+x) \Gamma(1+y)}{\Gamma(1+x+y)} \cdot e^{T y}
$$

and

$$
\sum_{m=0}^{\infty} P^{(x)}(\mathbf{k}_{+}, \underbrace{1, \ldots, 1}_{m} ; T) y^{m}=\sum_{m=0}^{\infty}(-1)^{m} \zeta^{(x)}\left(\mathbf{k} \circledast(\underbrace{1, \ldots, 1}_{m+1})^{\star}\right) y^{m} \cdot \frac{\Gamma(1+x) \Gamma(1+y)}{\Gamma(1+x+y)} \cdot e^{T y}
$$

for non-empty $\mathbf{k}$.

Proof. 1) To show (2.4), recall the identity (Lemma 5.1 in [5])

$$
\sum_{m=0}^{\infty}[\underbrace{1, \ldots, 1}_{m}] y^{m}=\exp _{*}\left(\sum_{n=1}^{\infty} \frac{(-1)^{n-1}}{n}[n] y^{n}\right)
$$

in $\mathcal{R}_{*}[[y]]$, where $\mathcal{R}_{*}$ is the space of formal $\mathbb{Q}$-linear combinations of indices equipped with the stuffle product $*$ (which is commutative and associative). Applying the $\zeta_{*}^{(x)}$-regularization on both sides, we obtain

$$
\sum_{m=0}^{\infty} Z_{*}^{(x)}(\underbrace{1, \ldots, 1}_{m} ; T) y^{m}=\exp \left(\sum_{n=2}^{\infty} \frac{(-1)^{n-1}}{n} \zeta^{(x)}(n) y^{n}\right) e^{T y} .
$$

We show that the identity

$$
\exp \left(\sum_{n=2}^{\infty} \frac{(-1)^{n-1}}{n} \zeta^{(x)}(n) y^{n}\right)=\frac{\Gamma(1+x) e^{\psi(1+x) y}}{\Gamma(1+x+y)}
$$

holds. The both sides become 1 when $y=0$, and the logarithmic derivative with respect to $y$ of the left-hand side is

$$
\sum_{n=2}^{\infty}(-1)^{n-1} \zeta^{(x)}(n) y^{n-1}
$$

which is equal to that of the right-hand side because $\zeta^{(x)}(n)=(-1)^{n} \psi^{(n-1)}(1+x) /(n-1)$ ! for $n \geq 2$. This proves (2.4).

For (2.5), we start with the identity

$$
[\mathbf{k}_{+}, \underbrace{1, \ldots, 1}_{m}]=\sum_{i=0}^{m}(-1)^{m-i}\left[\mathbf{k} \circledast(\underbrace{1, \ldots, 1}_{m+1-i})^{\star}\right] *[\underbrace{1, \ldots, 1}_{i}]
$$

in $\mathcal{R}_{*}$ (the case $\mathbf{l}=(\underbrace{1, \ldots, 1}_{m})$ of $\left(A_{*}\right)$ in Lemma 5.2 in [5]). From this we obtain by taking the $\zeta_{*}^{(x)}$-regularization and by forming the generating series

$$
\sum_{m=0}^{\infty} Z_{*}^{(x)}(\mathbf{k}_{+}, \underbrace{1, \ldots, 1}_{m} ; T) y^{m}=\sum_{m=0}^{\infty}(-1)^{m} \zeta^{(x)}\left(\mathbf{k} \circledast(\underbrace{1, \ldots, 1}_{m+1})^{\star}\right) y^{m} \cdot \sum_{i=0}^{\infty} Z_{*}^{(x)}(\underbrace{1, \ldots, 1}_{i} ; T) y^{i} .
$$


By (2.4), we obtain (2.5).

2) To show (2.6), we first prove a lemma.

Lemma 2.4. For any integer $m \geq 1$, it holds

$$
\mathrm{Li}_{\underbrace{(x) \ldots, 1}_{m}}^{(x)}(t)=\sum_{j=0}^{m}(-1)^{m-j} \int_{0}^{t} \underbrace{\frac{d u}{1-u} \cdots \frac{d u}{1-u} \frac{1-u^{x}}{1-u} d u}_{m-j} \cdot \frac{(-\log (1-t))^{j}}{j !} .
$$

The integral on the right is an abbreviated form of the iterated integral

$$
\int_{0}^{t} \underbrace{\frac{d u}{1-u} \cdots \frac{d u}{1-u} \frac{1-u^{x}}{1-u} d u}_{m-j}=\int_{0<u_{1}<\cdots<u_{m-j}<t} \frac{d u_{1}}{1-u_{1}} \cdots \frac{d u_{m-j-1}}{1-u_{m-j-1}} \frac{1-u_{m-j}^{x}}{1-u_{m-j}} d u_{m-j}
$$

and is regarded as 1 when $j=m$.

Proof. We start from the expression (which follows from (2.1))

$$
\mathrm{Li}_{\underbrace{1, \ldots, 1}_{m}}(t)-\mathrm{Li}_{\underbrace{1, \ldots, 1}_{m}}^{(x)}(t)=\int_{0}^{t} \frac{1-u^{x}}{1-u} d u \underbrace{\frac{d u}{1-u} \cdots \frac{d u}{1-u}}_{m-1}=I_{t}(
$$

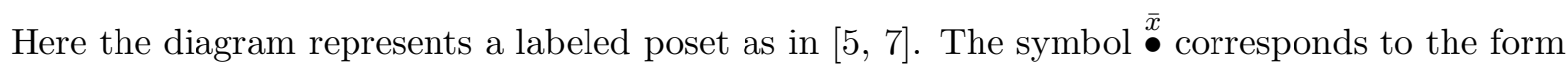
$\left(1-u^{x}\right) d u /(1-u)$. In [7], we used $\bullet^{x}$ instead, but we reserve this notation for $u^{x} d u /(1-u)$ in the later computation to show (2.7). Moreover, notice that we are considering the integral from 0 to $t$, represented by the symbol $I_{t}$. Then we compute as

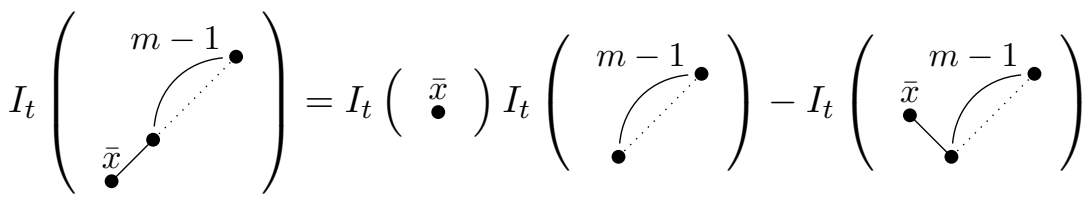

$$
\begin{aligned}
& =\cdots \\
& =\sum_{j=0}^{m-1}(-1)^{m-1-j} I_{t}(\stackrel{\bar{x}}{\bullet} \overbrace{}^{m}) I_{t}\left({ }^{j}\right),
\end{aligned}
$$

which gives (2.8) since

$$
I_{t}(\overbrace{}^{j})=\operatorname{Li}_{\underbrace{1, \ldots, 1}}(t)=\frac{(-\log (1-t))^{j}}{j !} \text {. }
$$

For any $l>0$, the value of the integral

$$
\int_{0}^{t} \underbrace{\frac{d u}{1-u} \cdots \frac{d u}{1-u} \frac{1-u^{x}}{1-u} d u}_{l}
$$


converges as $t \rightarrow 1$, and the remainder is estimated as

$$
\begin{aligned}
& \int_{0}^{1} \underbrace{\frac{d u}{1-u} \cdots \frac{d u}{1-u} \frac{1-u^{x}}{1-u} d u}_{l}-\int_{0}^{t} \underbrace{\frac{d u}{1-u} \cdots \frac{d u}{1-u} \frac{1-u^{x}}{1-u} d u}_{l} \\
& =\int_{t}^{1} \underbrace{}_{\underbrace{i_{1, \ldots, 1}}_{l-1}}(u) \frac{1-u^{x}}{1-u} d u=O\left((1-t) \log ^{l-1}(1-t)\right) \quad \text { as } t \rightarrow 1 .
\end{aligned}
$$

Indeed, $\left(1-u^{x}\right) /(1-u)$ is bounded by a constant and the estimate

$$
\int_{t}^{1} \operatorname{Li}_{\underbrace{1, \ldots, 1}_{l-1}}(u) d u=O\left((1-t) \log ^{l-1}(1-t)\right)
$$

is shown by induction on $l$. Moreover, we have

$$
\begin{aligned}
\int_{0}^{1} \underbrace{\frac{d u}{1-u} \cdots \frac{d u}{1-u} \frac{1-u^{x}}{1-u} d u}_{l} & =\sum_{n=1}^{\infty}(-1)^{n-1} x^{n} \int_{0<u_{1}<\cdots<u_{l}<1} \frac{d u_{1}}{1-u_{1}} \cdots \frac{d u_{l}}{1-u_{l}} \frac{1}{n !}\left(\int_{u_{l}}^{1} \frac{d v}{v}\right)^{n} \\
& =\sum_{n=1}^{\infty}(-1)^{n-1} x^{n} \int_{0}^{1} \underbrace{\frac{d u}{1-u} \cdots \frac{d u}{1-u}}_{l} \underbrace{\frac{d v}{v} \cdots \frac{d v}{v}}_{n} \\
& =\sum_{n=1}^{\infty}(-1)^{n-1} \zeta(\underbrace{1, \ldots, 1}_{l-1}, n+1) x^{n},
\end{aligned}
$$

and therefore we obtain

$$
P^{(x)}(\underbrace{1, \ldots, 1}_{m} ; T)=\sum_{j=0}^{m}(-1)^{m-j}\left(\sum_{n=1}^{\infty}(-1)^{n-1} \zeta(\underbrace{1, \ldots, 1}_{m-j-1}, n+1) x^{n}\right) \frac{T^{j}}{j !}
$$

from Lemma 2.4 and the definition of $P^{(x)}(\mathbf{k} ; T)$ (Proposition 2.1).

The generating series of

$$
\sum_{n=1}^{\infty}(-1)^{n-1} \zeta(\underbrace{1, \ldots, 1}_{l-1}, n+1) x^{n}
$$

is given by the well-known formula

$$
1+\sum_{l=1}^{\infty}(-1)^{l} \sum_{n=1}^{\infty}(-1)^{n-1} \zeta(\underbrace{1, \ldots, 1}_{l-1}, n+1) x^{n} y^{l}=\frac{\Gamma(1+x) \Gamma(1+y)}{\Gamma(1+x+y)},
$$

and thus (2.6) is obtained.

Now we define

$$
F(\underbrace{1, \ldots, 1}_{l} ; x):= \begin{cases}\sum_{n=1}^{\infty}(-1)^{n-1} \zeta(\underbrace{1, \ldots, 1}_{l-1}, n+1) x^{n} & (l>0), \\ 1 & (l=0),\end{cases}
$$


hence the above formula and the equation (2.10) can be written, respectively, as

$$
\sum_{l=0}^{\infty}(-1)^{l} F(\underbrace{1, \ldots, 1}_{l} ; x) y^{l}=\frac{\Gamma(1+x) \Gamma(1+y)}{\Gamma(1+x+y)}
$$

and

$$
P^{(x)}(\underbrace{1, \ldots, 1}_{m} ; T)=\sum_{j=0}^{m}(-1)^{m-j} F(\underbrace{1, \ldots, 1}_{m-j} ; x) \frac{T^{j}}{j !} .
$$

Note that, by the duality relation $\zeta(\underbrace{1, \ldots, 1}_{l-1}, n+1)=\zeta(\underbrace{1, \ldots, 1}_{n-1}, l+1)$, the function (2.11) coincides with the Kawashima function (3.1) for the index set $\mathbf{k}=(\underbrace{1, \ldots, 1}_{l})$. The identity

$$
\int_{0}^{1} \underbrace{\frac{d u}{1-u} \cdots \frac{d u}{1-u} \frac{1-u^{x}}{1-u} d u}_{l}=F(\underbrace{1, \ldots, 1}_{l} ; x)
$$

obtained above is a special case of [7, Proposition 2.8]. However, we should keep in mind that we adopt a different definition for the Kawashima function.

We now proceed to the proof of (2.7). Again we use the poset diagram for the iterated integral as in the proof of Lemma 2.4. As remarked before, the symbol $\stackrel{x}{\bullet}$ represents the form $u^{x} d u /(1-u)$. Recalling the integral expression (2.1), we compute

$$
\begin{aligned}
& \mathrm{Li}_{(\mathbf{k}_{+}, \underbrace{1, \ldots, 1}_{m})}^{(x)}(t)=I_{t} \\
& =I_{t}\left(\mathrm{k}^{0}\right) I_{t}\left(I_{t}^{m}\right. \\
& =\cdots \\
& =\sum_{j=0}^{m}(-1)^{m-j} I_{t}\left(\mathbf{k} \bullet I^{m-j}\right)
\end{aligned}
$$

We derive from this that

$$
P^{(x)}(\mathbf{k}_{+}, \underbrace{1, \ldots, 1}_{m} ; T)=\sum_{j=0}^{m}(-1)^{m-j} I(\mathbf{k})^{m-j} \frac{T^{j}}{j !},
$$

because the estimate

$$
\begin{aligned}
& I(\mathbf{k})^{m-j}-I_{t}(\underbrace{m-j}_{m-j}) \\
& =\int_{t}^{1} \operatorname{Li}_{\mathbf{k}}^{(x)}(u) \underbrace{}_{\underbrace{}_{1, \ldots, 1}}(u) \frac{d u}{u}=O\left((1-t) \log ^{p}(1-t)\right) \quad(\text { for some } p>0)
\end{aligned}
$$


follows from (2.9). The identity (2.7) will be a consequence of the following lemma.

Lemma 2.5. For $l \geq 0$, we have

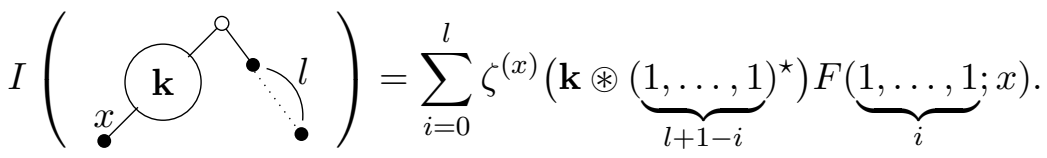

Proof. Let $I^{(x)}\left(\mathbf{k}_{+} ; l\right)$ denote the left-hand side. We also set $\mathbf{k}=\left(k_{1}, \ldots, k_{r}\right)$. Then we have

$$
\begin{aligned}
& I^{(x)}\left(\mathbf{k}_{+} ; l\right)=\int_{u_{0}>u_{1}>\cdots>u_{l}} \operatorname{Li}_{\mathbf{k}}^{(x)}\left(u_{0}\right) \frac{d u_{0}}{u_{0}} \frac{d u_{1}}{1-u_{1}} \cdots \frac{d u_{l}}{1-u_{l}} \\
& =\int_{u_{0}>u_{1}>\cdots>u_{l}} \sum_{0<m_{1}<\cdots<m_{r}} \frac{u_{0}^{m_{r}+x}}{\left(m_{1}+x\right)^{k_{1}} \cdots\left(m_{r}+x\right)^{k_{r}}} \frac{d u_{0}}{u_{0}} \frac{d u_{1}}{1-u_{1}} \cdots \frac{d u_{l}}{1-u_{l}} \\
& =\int_{u_{1}>\cdots>u_{l}} \sum_{0<m_{1}<\cdots<m_{r}} \frac{1-u_{1}^{m_{r}+x}}{\left(m_{1}+x\right)^{k_{1}} \cdots\left(m_{r}+x\right)^{k_{r}+1}} \frac{d u_{1}}{1-u_{1}} \cdots \frac{d u_{l}}{1-u_{l}} .
\end{aligned}
$$

Here each variable $u_{i}$ is in the interval $(0,1)$ and we omit the bounds 0 and 1 in the notation of the integral. Now we split the last integral into two by writing

$$
1-u_{1}^{m_{r}+x}=1-u_{1}^{x}+u_{1}^{x}-u_{1}^{m_{r}+x}=\left(1-u_{1}^{x}\right)+u_{1}^{x}\left(1-u_{1}^{m_{r}}\right),
$$

and thus obtain

$$
\begin{aligned}
I^{(x)}\left(\mathbf{k}_{+} ; l\right) & =\int_{u_{1}>\cdots>u_{l}} \sum_{0<m_{1}<\cdots<m_{r}} \frac{1}{\left(m_{1}+x\right)^{k_{1}} \cdots\left(m_{r}+x\right)^{k_{r}+1}} \frac{1-u_{1}^{x}}{1-u_{1}} d u_{1} \frac{d u_{2}}{1-u_{2}} \cdots \frac{d u_{l}}{1-u_{l}} \\
& +\int_{u_{1}>\cdots>u_{l}} \sum_{0<m_{1}<\cdots<m_{r}} \frac{1}{\left(m_{1}+x\right)^{k_{1}} \cdots\left(m_{r}+x\right)^{k_{r}+1}} \frac{u_{1}^{x}\left(1-u_{1}^{m_{r}}\right)}{1-u_{1}} d u_{1} \frac{d u_{2}}{1-u_{2}} \cdots \frac{d u_{l}}{1-u_{l}} .
\end{aligned}
$$

The infinite series in the first integral is nothing but $\zeta^{(x)}\left(\mathbf{k}_{+}\right)=\zeta^{(x)}\left(\mathbf{k} \circledast(1)^{\star}\right)$ which is independent of the integral variables there, and the integral equals $F(\underbrace{1, \ldots, 1}_{l} ; x)$ by (2.14). So this term $\zeta^{(x)}\left(\mathbf{k} \circledast(1)^{\star}\right) F(\underbrace{1, \ldots, 1}_{l} ; x)$ is the term for $i=l$ on the right of the lemma.

As for the second integral, we integrate with respect to $u_{1}$ after writing $\frac{1-u_{1}^{m_{r}}}{1-u_{1}}=\sum_{n_{1}=1}^{m_{r}} u_{1}^{n_{1}-1}$ to obtain

$$
\begin{aligned}
& \int_{u_{1}>\cdots>u_{l}} \sum_{0<m_{1}<\cdots<m_{r}} \frac{1}{\left(m_{1}+x\right)^{k_{1}} \cdots\left(m_{r}+x\right)^{k_{r}+1}} \sum_{n_{1}=1}^{m_{r}} u_{1}^{x+n_{1}-1} d u_{1} \frac{d u_{2}}{1-u_{2}} \cdots \frac{d u_{l}}{1-u_{l}} \\
&= \sum_{u_{2}>\cdots>u_{l}} \sum_{0<m_{1}<\cdots<m_{r} \geq n_{1} \geq 1} \frac{1-u_{2}^{n_{1}+x}}{\left(m_{1}+x\right)^{k_{1}} \cdots\left(m_{r}+x\right)^{k_{r}+1}\left(n_{1}+x\right)} \frac{d u_{2}}{1-u_{2}} \cdots \frac{d u_{l}}{1-u_{l}} \\
&= \sum_{u_{2}>\cdots>u_{l}} \sum_{0<m_{1}<\cdots<m_{r}=n_{0} \geq n_{1} \geq 1} \frac{1-u_{2}^{n_{1}+x}}{\left(m_{1}+x\right)^{k_{1}} \cdots\left(m_{r}+x\right)^{k_{r}}\left(n_{0}+x\right)\left(n_{1}+x\right)} \\
& \frac{d u_{2}}{1-u_{2}} \cdots \frac{d u_{l}}{1-u_{l}} .
\end{aligned}
$$


As before, writing $1-u_{2}^{n_{1}+x}=\left(1-u_{2}^{x}\right)+u_{2}^{x}\left(1-u_{2}^{n_{1}}\right)$ and splitting the integral into two, we get from the first term $\zeta^{(x)}\left(\mathbf{k} \circledast(1,1)^{\star}\right) \cdot F(\underbrace{1, \ldots, 1}_{l-1} ; x)$ which is the $i=l-1$ term in the lemma. We integrate the second term with respect to $u_{2}$ after writing $\frac{1-u_{2}^{n_{1}}}{1-u_{2}}=\sum_{n_{2}=1}^{n_{1}} u_{2}^{n_{2}-1}$ and the same procedure continues to obtain the desired formula. The proof of the lemma is complete.

Proof of (2.7). By the equation (2.15) and Lemma 2.5, we have

$$
\begin{aligned}
& \sum_{m=0}^{\infty} P^{(x)}(\mathbf{k}_{+}, \underbrace{1, \ldots, 1}_{m} ; T) y^{m} \\
& =\sum_{m=0}^{\infty} \sum_{j=0}^{m}(-1)^{m-j} \sum_{i=0}^{m-j} \zeta^{(x)}\left(\mathbf{k} \circledast(\underbrace{1, \ldots, 1}_{m-j+1-i})^{\star}\right) F(\underbrace{1, \ldots, 1}_{i} ; x) \frac{T^{j}}{j !} y^{m} \\
l:=m-j-i & \sum_{l, i, j=0}^{\infty}(-1)^{l+i} \zeta^{(x)}\left(\mathbf{k} \circledast(\underbrace{1, \ldots, 1}_{l+1})^{\star}\right) F(\underbrace{1, \ldots, 1}_{i} ; x) \frac{T^{j}}{j !} y^{l+i+j} .
\end{aligned}
$$

Thus the equation (2.7) follows from (2.12).

Proof of Theorem 2.2. Replacing $T$ in (2.5) by $T-\gamma-\psi(1+x)$, and applying the map $\rho$ on both sides, we see that the right-hand side becomes that of (2.7), and we conclude the theorem.

\section{The Kawashima function and Kawashima's relation}

For a non-empty index set $\mathbf{k}$, we define the Kawashima function $F(\mathbf{k} ; x)$ by

$$
F(\mathbf{k} ; x):=\sum_{m=1}^{\infty}(-1)^{m-1} \zeta\left((\underbrace{1, \ldots, 1}_{m}) \circledast\left(\mathbf{k}^{\vee}\right)^{\star}\right) x^{m} .
$$

Here $\mathbf{k}^{\vee}$ denotes the Hoffman dual of $\mathbf{k}$ (see [5, Definition 6.3]). Originally, Kawashima [6] defined $F(\mathbf{k} ; x)$ via the Newton series and obtained this expression as a Taylor expansion at $x=0$. We remark here that, because of the duality $\zeta((\underbrace{1, \ldots, 1}_{m}) \circledast(1))=\zeta(\underbrace{1, \ldots, 1}_{m-1}, 2)=\zeta(m+1)$, we have $F(1 ; x)=\psi(1+x)+\gamma$. Recall that $x$ is a real variable with $|x|<1$ throughout the present paper.

Our main result in this section is the following.

Theorem 3.1. For any $\mathbf{k}=\left(k_{1}, \ldots, k_{r}\right) \in \mathbb{N}^{r}$, we have

$$
F(\mathbf{k} ; x)=\sum_{j=0}^{r}(-1)^{r-j} Z_{*}^{\star}\left(k_{1}, \ldots, k_{j} ; T\right) Z_{*}^{(x)}\left(k_{r}, \ldots, k_{j+1} ; T-\gamma-\psi(1+x)\right) .
$$

Here, $Z_{*}^{\star}(\mathbf{k} ; T):=Z_{*}\left(\mathbf{k}^{\star} ; T\right)$ is the stuffle regularized polynomial for the multiple zeta-star values (see [5]). Let $\bar{*}$ be the stuffle product for $\zeta^{\star}(\mathbf{k})$ 's, that is, the product so defined that we have $\zeta^{\star}(\mathbf{k}) \zeta^{\star}(\mathbf{l})=\zeta^{\star}(\mathbf{k} \bar{*} \mathbf{l})$ (also see [5] for the definition). Then, since $Z_{*}^{\star}$ and $Z_{*}^{(x)}$ satisfy $\bar{*}$ and $*$-product rules respectively, we conclude by the standard argument using the Hopf algebra structure on $\mathcal{R}_{*}$ (see [1]), that $F(\mathbf{k} ; x)$ satisfies the $\bar{*}$-product rule (here again we extend $F(-; x)$ by linearity to the formal sum of indices)

$$
F(\mathbf{k} ; x) F(\mathbf{l} ; x)=F(\mathbf{k} \bar{*} \mathbf{l} ; x),
$$


and thereby obtain the following Kawashima's relations by comparing the coefficients on both sides of (3.2).

Corollary 3.2. For any $m \geq 1$ and any non-empty index sets $\mathbf{k}$ and $\mathbf{l}$, we have

$$
\sum_{\substack{p+q=m \\ p, q \geq 1}} \zeta\left((\underbrace{1, \ldots, 1}_{p}) \circledast\left(\mathbf{k}^{\vee}\right)^{\star}\right) \zeta\left((\underbrace{1, \ldots, 1}_{q}) \circledast\left(\mathbf{l}^{\vee}\right)^{\star}\right)=-\zeta\left((\underbrace{1, \ldots, 1}_{m}) \circledast\left((\mathbf{k} \bar{*} \mathbf{l})^{\vee}\right)^{\star}\right) \quad(\forall m \geq 1) .
$$

The next proposition is the key to our proof of the theorem. To state it, we need a variant $F(\mathbf{k} ; x ; t)$ of the Kawashima function with an additional parameter $t(0<t<1)$ defined by

$$
F(\mathbf{k} ; x ; t)=\sum_{m=1}^{\infty}(-1)^{m-1} \hat{\zeta}\left((\underbrace{1, \ldots, 1}_{m}) ; \mathbf{k}^{\vee} ; 1-t\right) x^{m},
$$

where for $\mathbf{k}=\left(k_{1}, \ldots, k_{r}\right)$ and $\mathbf{l}=\left(l_{1}, \ldots, l_{s}\right)$ we set

$$
\hat{\zeta}(\mathbf{k} ; \mathbf{l} ; t):=\sum_{0<m_{1}<\cdots<m_{r}=n_{s} \geq \cdots \geq n_{1}>0} \frac{t^{n_{1}}}{m_{1}^{k_{1}} \cdots m_{r}^{k_{r}} n_{1}^{l_{1}} \cdots n_{s}^{l_{s}}} .
$$

In particular, the value $\hat{\zeta}(\mathbf{k} ; \mathbf{l} ; 1)$ at $t=1$ is equal to $\zeta\left(\mathbf{k} \circledast \mathbf{l}^{\star}\right)$. Note that the power of $t$ in the sum on the right of the definition (3.3) is $n_{1}$, not the outer $n_{s}$. In the following we use the formula for the derivative

$$
\frac{d}{d t} \hat{\zeta}(\mathbf{k} ; \mathbf{l} ; t)= \begin{cases}\frac{1}{t} \hat{\zeta}\left(\mathbf{k} ;\left(l_{1}-1, l_{2}, \ldots, l_{s}\right) ; t\right) & \left(l_{1}>1\right), \\ \frac{1}{1-t}\left[\zeta\left(\mathbf{k} \circledast\left(l_{2}, \ldots, l_{s}\right)\right)-\hat{\zeta}\left(\mathbf{k} ;\left(l_{2}, \ldots, l_{s}\right) ; t\right)\right] & \left(l_{1}=1\right)\end{cases}
$$

when $l_{1}+\cdots+l_{s} \geq 2$, which follows easily from the definition (3.3). Note that, when $\mathbf{l}=(1)$, we have $\hat{\zeta}(\mathbf{k} ; 1 ; t)=\mathrm{Li}_{k_{1}, \ldots, k_{r}+1}(t)$ and its derivative is well-known. Using these, we obtain

$$
\begin{aligned}
\frac{\partial}{\partial t} F & \left(k_{r}, \ldots, k_{1} ; x ; t\right) \\
& = \begin{cases}-\frac{1}{1-t} F\left(k_{r-1}, \ldots, k_{1} ; x ; t\right) & \left(k_{r}=1\right), \\
\frac{1}{t}\left[F\left(k_{r}-1, k_{r-1}, \ldots, k_{1} ; x ; t\right)-F\left(k_{r}-1, k_{r-1}, \ldots, k_{1} ; x\right)\right] & \left(k_{r}>1\right),\end{cases}
\end{aligned}
$$

when $k_{1}+\cdots+k_{r}>1$. For the case of weight one, we have

$$
\begin{aligned}
\frac{\partial}{\partial t} F(1 ; x, t) & =\frac{1}{1-t} \sum_{m=1}^{\infty}(-1)^{m} \operatorname{Li}_{\underbrace{1, \ldots, 1}_{m}}(1-t) x^{m}=\frac{1}{1-t} \sum_{m=1}^{\infty} \frac{\log ^{m} t}{m !} x^{m} \\
& =\frac{t^{x}-1}{1-t} .
\end{aligned}
$$

Proposition 3.3. For a non-empty index set $\mathbf{k}=\left(k_{1}, \ldots, k_{r}\right)$, we have

$$
\mathrm{Li}_{\mathbf{k}}^{(x)}(t)+(-1)^{r} F(\overleftarrow{\mathbf{k}} ; x ; t)=\sum_{j=0}^{r}(-1)^{j} F\left(k_{j}, \ldots, k_{1} ; x\right) \mathrm{Li}_{k_{j+1}, \ldots, k_{r}}(t)
$$

where $\overleftarrow{\mathbf{k}}:=\left(k_{r}, \ldots, k_{1}\right)$ 
Proof. We proceed by induction on the weight $k:=k_{1}+\cdots+k_{r}$. When the weight is 1 , the left-hand side is $\operatorname{Li}_{1}^{(x)}(t)-F(1 ; x ; t)$ and the right-hand side is $\operatorname{Li}_{1}(t)-F(1 ; x)$. Both of these have the value $-F(1 ; x)$ at $t=0$. From the differential formula (3.5) together with

$$
\frac{\partial}{\partial t} \operatorname{Li}_{1}^{(x)}(t)=\frac{t^{x}}{1-t}
$$

we see that the derivatives with respect to $t$ of both sides coincide. Hence the case $k=1$ is proved.

Assume the weight $k$ is greater than 1 and the formula is valid for lower weights. If $k_{r}=1$, then we have

$$
\frac{\partial}{\partial t}(\text { L.H.S. })=\frac{1}{1-t} \operatorname{Li}_{k_{1}, \ldots, k_{r-1}}^{(x)}(t)+\frac{(-1)^{r+1}}{1-t} F\left(k_{r-1}, \ldots, k_{1} ; x ; t\right)
$$

and

$$
\left.\frac{\partial}{\partial t} \text { (R.H.S. }\right)=\sum_{j=0}^{r-1}(-1)^{j} F\left(k_{j}, \ldots, k_{1} ; x\right) \cdot \frac{1}{1-t} \operatorname{Li}_{k_{j+1}, \ldots, k_{r-1}}(t) .
$$

The last expression is equal to the previous one by the induction hypothesis and we are done. If $k_{r}>1$, then we have

$$
\begin{aligned}
\frac{\partial}{\partial t}(\text { L.H.S. })= & \frac{1}{t} \operatorname{Li}_{k_{1}, \ldots, k_{r-1}, k_{r}-1}^{(x)}(t) \\
& +\frac{(-1)^{r}}{t}\left[F\left(k_{r}-1, k_{r-1}, \ldots, k_{1} ; x ; t\right)-F\left(k_{r}-1, k_{r-1}, \ldots, k_{1} ; x\right)\right]
\end{aligned}
$$

and

$$
\left.\frac{\partial}{\partial t} \text { (R.H.S. }\right)=\sum_{j=0}^{r-1}(-1)^{j} F\left(k_{j}, \ldots, k_{1} ; x\right) \cdot \frac{1}{t} \operatorname{Li}_{k_{j+1}, \ldots, k_{r-1}, k_{r}-1}(t),
$$

which are equal by the induction hypothesis.

Proof of Theorem 3.1. Considering the asymptotic behavior of (3.6) as $t \rightarrow 1$, we obtain

$$
P^{(x)}(\mathbf{k} ; T)=\sum_{j=0}^{r}(-1)^{j} F\left(k_{j}, \ldots, k_{1} ; x\right) Z_{\mathrm{II}}\left(k_{j+1}, \ldots, k_{r} ; T\right) .
$$

Applying the map $\rho^{-1}$ on both sides and using Theorem 2.2, we obtain

$$
Z_{*}^{(x)}(\mathbf{k} ; T-\gamma-\psi(x+1))=\sum_{j=0}^{r}(-1)^{j} F\left(k_{j}, \ldots, k_{1} ; x\right) Z_{*}\left(k_{j+1}, \ldots, k_{r} ; T\right) .
$$

From this, we obtain Theorem 3.1 (and vice versa) by using the 'andipode relation'

$$
\sum_{j=0}^{r}(-1)^{r-j} Z_{*}^{\star}\left(k_{1}, \ldots, k_{j} ; T\right) Z_{*}\left(k_{r}, \ldots, k_{j+1} ; T\right)= \begin{cases}1 & (r=0), \\ 0 & (r>0)\end{cases}
$$

coming from the Hopf algebra structure on $\mathcal{R}_{*}$. 


\section{Various remarks}

1. The right-hand side of Theorem 3.1 is independent of $T$, so that we may set $T=0$ or $T=\gamma+\psi(1+x)$ to obtain

$$
F(\mathbf{k} ; x)=\sum_{j=0}^{r}(-1)^{r-j} \zeta_{*}^{\star}\left(k_{1}, \ldots, k_{j}\right) Z_{*}^{(x)}\left(k_{r}, \ldots, k_{j+1} ;-\gamma-\psi(1+x)\right)
$$

or

$$
F(\mathbf{k} ; x)=\sum_{j=0}^{r}(-1)^{r-j} Z_{*}^{\star}\left(k_{1}, \ldots, k_{j} ; \gamma+\psi(1+x)\right) \zeta_{*}^{(x)}\left(k_{r}, \ldots, k_{j+1}\right),
$$

or from (3.7)

$$
\zeta_{*}^{(x)}(\mathbf{k})=\sum_{j=0}^{r}(-1)^{j} F\left(k_{j}, \ldots, k_{1} ; x\right) Z_{*}\left(k_{j+1}, \ldots, k_{r} ; \gamma+\psi(x+1)\right) .
$$

In particular, if all $k_{i}$ are larger than 1 , no regularization is needed and we have

$$
\zeta^{(x)}\left(k_{1}, \ldots, k_{r}\right)=\sum_{j=0}^{r}(-1)^{j} F\left(k_{j}, \ldots, k_{1} ; x\right) \zeta\left(k_{j+1}, \ldots, k_{r}\right)
$$

and equivalently

$$
F\left(k_{1}, \ldots, k_{r} ; x\right)=\sum_{j=0}^{r}(-1)^{r-j} \zeta^{\star}\left(k_{1}, \ldots, k_{j}\right) \zeta^{(x)}\left(k_{r}, \ldots, k_{j+1}\right) .
$$

2. There is yet another route to reach Theorem 3.1, by adopting a different definition of the Kawashima function as a starting point.

For index sets $\mathbf{k}=\left(k_{1}, \ldots, k_{r}\right)$, define $F(\mathbf{k} ; x)$ inductively by

$$
F(\varnothing ; x)=1, \quad F(\mathbf{k} ; x)=\sum_{n=1}^{\infty}\left(\frac{F\left(\mathbf{k}^{\prime} ; n\right)}{n^{k_{r}}}-\frac{F\left(\mathbf{k}^{\prime} ; n+x\right)}{(n+x)^{k_{r}}}\right),
$$

where $\mathbf{k}^{\prime}=\left(k_{1}, \ldots, k_{r-1}\right)$. Then we can show that the identity

$$
\begin{aligned}
& F\left(k_{1}, \ldots, k_{r} ; x\right) \\
& =\lim _{N \rightarrow \infty} \sum_{j=1}^{r}(-1)^{r-j} \sum_{\substack{0<n_{1} \leq \cdots \leq n_{j}<N \\
n_{j}>n_{j+1}>\cdots>n_{r}>0}} \frac{1}{n_{1}^{k_{1}} \ldots n_{j-1}^{k_{j-1}}}\left(\frac{1}{n_{j}^{k_{j}}}-\frac{1}{\left(n_{j}+x\right)^{k_{j}}}\right) \\
& \times \frac{1}{\left(n_{j+1}+x\right)^{k_{j+1}} \ldots\left(n_{r}+x\right)^{k_{r}}}
\end{aligned}
$$

holds. For a fixed $N$, we see that the (finite) sum on the right is equal to

$$
\sum_{j=0}^{r}(-1)^{r-j} \zeta_{N}^{\star}\left(k_{1}, \ldots, k_{j}\right) \zeta_{N}^{(x)}\left(k_{r}, \ldots, k_{j+1}\right) .
$$

Noting the asymptotic behaviors

$$
\zeta_{N}^{\star}\left(k_{1}, \ldots, k_{j}\right)=Z_{*}^{\star}\left(k_{1}, \ldots, k_{j} ; \log N+\gamma\right)+O\left(N^{-1} \log ^{p} N\right) \quad(\exists p>0)
$$


and

$$
\zeta_{N}^{(x)}\left(k_{r}, \ldots, k_{j+1}\right)=Z_{*}^{(x)}\left(k_{r}, \ldots, k_{j+1} ; \log N-\psi(1+x)\right)+O\left(N^{-1} \log ^{p} N\right) \quad(\exists p>0),
$$

we conclude that the polynomial

$$
\sum_{j=0}^{r}(-1)^{r-j} Z_{*}^{\star}\left(k_{1}, \ldots, k_{j} ; T+\gamma\right) Z_{*}^{(x)}\left(k_{r}, \ldots, k_{j+1} ; T-\psi(1+x)\right)
$$

(obtained by replacing $\log N$ by $T$ ) is independent of $T$, and is equal to $F\left(k_{1}, \ldots, k_{r} ; x\right)$. By replacing $T$ by $T-\gamma$ we obtain Theorem 3.1 .

We note that a formula quite analogous to (4.1) for a variant of $F(\mathbf{k} ; x)$ (also defined inductively as above) has been obtained by K. Ihara and Y. Nakamura. We surmise that their function has an analogous expression in terms of some regularized polynomials as ours.

3. For any admissible index set $\mathbf{k}$, the Hurwitz multiple zeta value $\zeta^{(x)}(\mathbf{k})$ has a nice Taylor expansion at $x=0$ :

$$
\zeta^{(x)}(\mathbf{k})=\sum_{m=0}^{\infty} \zeta_{\amalg}(\mathbf{k}^{\dagger}, \underbrace{1, \ldots, 1}_{m}) x^{m} .
$$

Here, $\zeta_{\mathrm{II}}$ on the right is the shuffle regularized value and $\mathbf{k}^{\dagger}$ is the usual dual of $\mathbf{k}$ (in the notation of our paper, $\zeta_{\amalg}(\mathbf{k}^{\dagger}, \underbrace{1, \ldots, 1}_{m})$ equals $\left.P^{(0)}(\mathbf{k}^{\dagger}, \underbrace{1, \ldots, 1}_{m} ; 0)\right)$. This can be deduced by combining Propositon 3.9 and Theorem 2.5 in [4]. We may also start from the integral expression

$$
\left.\zeta^{(x)}(\mathbf{k})=I(x)\right)
$$

which is the equation (2.1) at $t=1$. Then we expand $u^{x}$ in the integral as

$$
u^{x}=\sum_{m=0}^{\infty} \frac{(\log u)^{m}}{m !} x^{m}=\sum_{m=0}^{\infty} \frac{(-1)^{m}}{m !}\left(\int_{u}^{1} \frac{d v}{v}\right)^{m} x^{m}=\sum_{m=0}^{\infty}(-1)^{m} \int_{u<v_{1}<\cdots<v_{m}<1} \frac{d v_{1}}{v_{1}} \cdots \frac{d v_{m}}{v_{m}} x^{m}
$$

to obtain

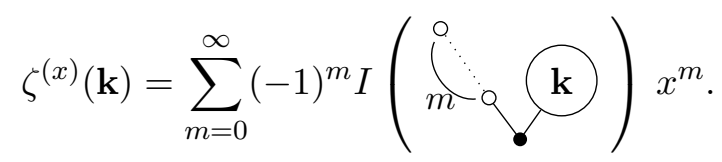

By the duality and the regularization formula [2, Prop. 8], we have

$$
I(\overbrace{m}^{0} \mathbf{k})=I\left(\mathbf{k}^{\dagger} \cdot \jmath^{m}\right)=(-1)^{m} \zeta_{\text {Шा }}(\mathbf{k}^{\dagger}, \underbrace{1, \ldots, 1}_{m}) .
$$

We should also note that, by the "integral-series identity" ([5, Th. 4.1]), we have

$$
\zeta_{\text {Шा }}(\mathbf{k}^{\dagger}, \underbrace{1, \ldots, 1}_{m})=(-1)^{m} \zeta\left(\left(\mathbf{k}^{\dagger}\right)_{-} \circledast(\underbrace{1, \ldots, 1}_{m+1})^{\star}\right),
$$

where $\left(\mathbf{k}^{\dagger}\right)_{-}$is the index obtained from $\mathbf{k}^{\dagger}$ by subtracting 1 from the last component. 


\section{References}

[1] M. E. Hoffman, Quasi-symmetric functions and $\bmod p$ multiple harmonic sums, Kyushu J. Math. 69 (2015), 345-366.

[2] K. Ihara, M. Kaneko and D. Zagier, Derivation and double shuffle relations for multiple zeta values, Compositio Math., 142 (2006), 307-338.

[3] M. Kaneko, An introduction to classical and finite multiple zeta values, Publications mathématiques de Besançon, 2019/1, 103-129.

[4] M. Kaneko and H. Tsumura, Multi-poly-Bernoulli numbers and related zeta functions, Nagoya Math. J. 232 (2018), 19-54.

[5] M. Kaneko and S. Yamamoto, A new integral-series identity of multiple zeta values and regularizations, Selecta Math., 24 (2018), 2499-2521.

[6] G. Kawashima, A class of relations among multiple zeta values, J. Number Theory, 129 (2009), $755-788$.

[7] S. Yamamoto, A note on Kawashima functions, Publications mathématiques de Besançon 2019/1, 151-163.

Masanobu Kaneko

Faculty of Mathematics, Kyushu University

744 Motooka, Nishi-ku, Fukuoka, 819-0395, JAPAN

e-mail: mkaneko@math.kyushu-u.ac.jp

$\mathrm{Ce} \mathrm{Xu}$

School of Mathematics and Statistics, Anhui Normal University

Wuhu 241000, People's Republic of China

e-mail: 2020008@ahnu.edu.cn

Shuji Yamamoto

Department of Mathematics, Faculty of Science and Technology, Keio University

3-14-1 Hiyoshi, Kohoku-ku, Yokohama, 223-8522, JAPAN

e-mail: yamashu@math.keio.ac.jp 